\title{
Wide Tuning Range Inversion-Mode Gated Varactor and Its Application on a 2-GHz VCO
}

\author{
Wallace Wong, Frankie Hui, Zhiheng Chen, Keqiang Shen ${ }^{\dagger}$, Jack Lau, Philip Chan, Ping Ko \\ Department of E.E.E., The Hong Kong University of Science \& Technology, Clear Water Bay, Kowloon, Hong Kong \\ ${ }^{\dagger}$ Currently on leave from Southeast University, P.R.C.
}

\begin{abstract}
This paper presents a novel inversion-mode three-terminal varactor with a capacitance ranging from $0.7 \mathrm{pF}$ to $2.3 \mathrm{pF}$, achieving a $\pm 53 \%$ variation in the capacitance. The new device is implemented in a $0.35-\mu \mathrm{m}$ standard CMOS process. The measured quality factor is 22 at $2 \mathrm{GHz}$. A 2-GHz VCO is designed with this new varactor and is able to achieve a tuning range of $320 \mathrm{MHz}$ with a maximum tuning gain of $220 \mathrm{MHz} / \mathrm{V}$.
\end{abstract}

\section{Introduction}

Being a key block of monolithic transceivers, fully integrated CMOS oscillators are of great interest. Even though they exhibit lower phase noise than ring oscillators, the integrated $L C$-tank oscillators have very limited tuning range. Wide tuning range accumulation-mode varactors with a tuning range of about $\pm 30 \%$ were proposed by R. Castello [1] and T. Soorapanth [2] in 1998. In this work, we present a new varactor, namely the gated varactor, which has a wider tuning range than the previous works. When implemented in a $0.35-\mu \mathrm{m}$ standard CMOS process, the varactor achieved a capacitance ranging of $\pm 53 \%$ with a nominal capacitance of $1.5 \mathrm{pF}$. A 2-GHz VCO implemented with the gated varactors has circuit simplicity over recently reported switched tuning VCO [3].

\section{The Varactor Structure}

The cross-sectional view of the varactor is shown in Fig. 1. The structure of the varactor is similar to a p-channel MOS transistor with the drain replaced by an $\mathrm{N}+$ region. The new varactor is a threeterminal device rather than a two-terminal device as in conventional varactor. The total capacitance is made up by the gate capacitance $C_{g}$ and the lateral junction capacitance $C_{p n}$. The total capacitance measured at the drain can be varied by varying the drain voltage and the gate voltage.

When a positive voltage is applied to the gate and the source is grounded. The total capacitance $\left(C_{\max }\right)$ is equal to the gate oxide capacitance plus the drain/source junction capacitance. The $C_{\max }$ of the gated varactor is larger than the $C_{\max }$ of the accumulation-mode varactor which is essentially the gate oxide capacitance.

When the gate is grounded and a positive voltage is applied at the drain, an inversion layer is formed beneath the gate and the region under the channel is further depleted by the drain due to the subsurface DIBL effect. As a result, the depletion region can extend further than the accumulation-mode varactor and hence achieving a minimum capacitance less than that of the accumulation-mode varactor. The variation of the depletion region with the drain voltage is simulated by MEDICI and shown in Fig. 2.

\section{Measurement Results}

The new varactor is implemented in a $0.35-\mu \mathrm{m}$ CMOS technology. The fabricated varactor consists of 100 segments each of size $5 \mu \mathrm{m} \times 1.6 \mu \mathrm{m}$. Fig. 3 is a plot of capacitance value versus the biasing voltages. The capacitance of the varactor increases from 0.8 $\mathrm{pF}$ to $2.25 \mathrm{pF}$ as the gate/drain voltage varies from $0 \mathrm{~V}$ to $2 \mathrm{~V}$. The variation covers $90 \%$ of the tuning range. This allows the varactor to be used in low-voltage VCO. Wide tuning range VCO can be achieved without increasing the power supply voltage.

The maximum quality factor of the varactor is measured to be 22 at 2-GHz. The measured quality factor is shown in Fig. 4.

\section{Circuit Design with the Gated Varactor}

A 2-GHz $\mathrm{VCO}$ is designed with the new varactor $\left(\mathrm{C}_{1}\right.$ and $\left.\mathrm{C}_{2}\right)$ which is shown in Fig. 5. In the VCO, the gate bias of the varactors is controlled via $\mathrm{V}_{\mathrm{ctrl}}$, and the drain of the varactors maintains a positive voltage by $\mathrm{V}_{\text {tune }}$ through the biasing circuit. As the control voltage $\left(\mathrm{V}_{\text {tune }}\right)$ increases, both potentials across the drain/source and drain/gate reduce.

Reducing the voltage across the drain/source, the depletion region underneath the channel shrinks, and hence increases the total capacitance. Reducing the voltage across the drain/gate, the device is driven from inversion mode to depletion mode and the total capacitance is increased.

Applying different ratio of voltage at the source and the gate can vary the sensitivity of the VCO. In our VCO, the sensitive reaches the maximum when the ratio is $1: 1$. The resultant tuning characteristic is shown by the dotted line of Fig. 6. The general tuning characteristic of the $\mathrm{VCO}$ at different biasing is shown in Fig. 6. The VCO is able to achieve a maximum sensitivity of 220 $\mathrm{MHz} / \mathrm{V}$ when $\mathrm{V}_{\text {ctrl }}$ and $\mathrm{V}_{\text {tune }}$ are configured to form a single tuning node.

\section{Conclusions}

A novel inversion-mode gated varactor demonstrated a wide tuning range of $\pm 53 \%$ at $1.5 \mathrm{pF}$. The implementation of gated varactors to the $2-\mathrm{GHz} L C$-tank VCO fabricated on $0.35-\mu \mathrm{m}$ CMOS technology showed a frequency tuning of $320 \mathrm{MHz}$. Fig. 7 shows the die photo of the VCO which measures $910 \mu \mathrm{m}$ by $740 \mu \mathrm{m}$ including the pads. The gated varactor has a compact layout size and can be easily incorporated with available fabrication technology with no extra process steps.

\section{Acknowledgments}

This project is partially funded by the Hong Kong RGC Earmarked Research Grant.

\section{References}

[1] R. Castello, P. Erratico, S. Manzini and F. Svelto, "A $\pm 30 \%$ Tuning Range Varactor Compatible with Future Scaled Technologies", 1998 Symposium on VLSI Circuits Digest of Technical Papers, pp. 34-35, June 1998

[2] T. Soorapanth, C. P. Yue, D. K. Shaeffer, T. H. Lee, S. S. Wong, "Analysis and optimization of accumulation-mode varactor for RF ICs", 1998 Symposium on VLSI Circuits Digest of Technical Papers, pp. 32-33, June 1998

[3] A. Kral, F. Behbahani, and A. A. Abidi, "RF-CMOS Oscillators with Switched Tuning", Proceedings of the IEEE 1998 Custom Integrated Circuit Conference, pp. 555-558, May 1998 


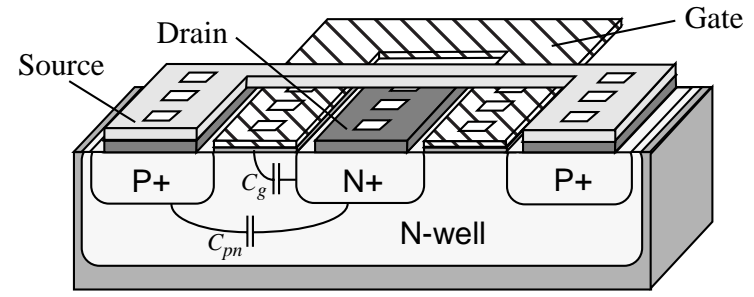

Fig. 1. Cross-section of the a P-type (N-body) gated varactor. $\mathrm{V}_{\mathrm{d}}$ and $\mathrm{V}_{\mathrm{g}}$ are the bias voltage applied to the "Drain" and "Gate" respectively.

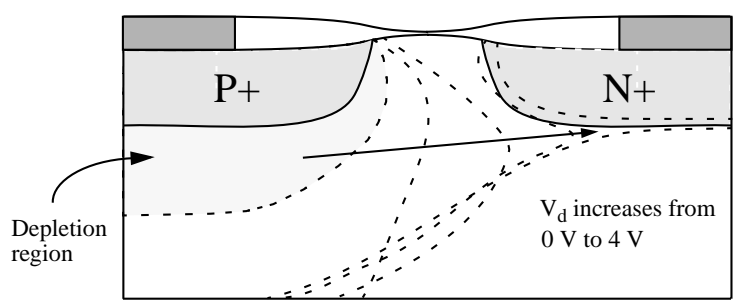

Fig. 2. MEDICI simulation of the variation of depletion region of the gated varactor upon increasing $\mathrm{V}_{\mathrm{d}}$ at a fixed gate voltage.

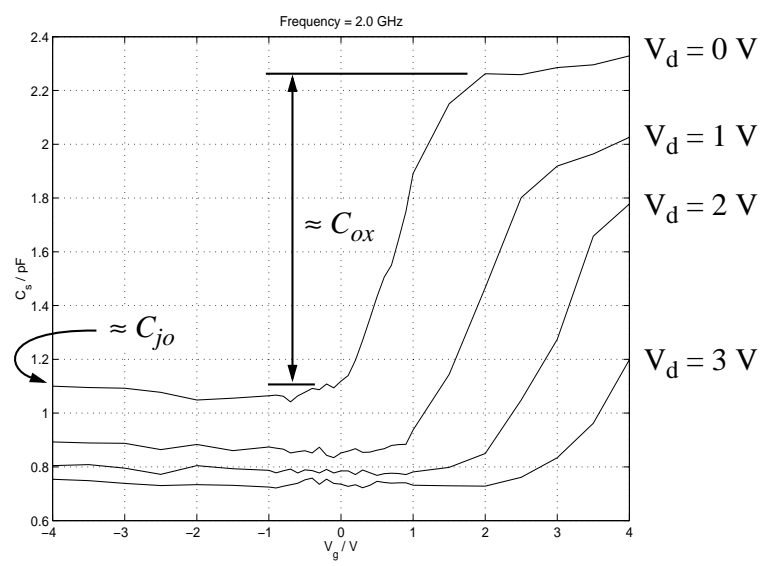

Fig. 3. The $\mathrm{C}-\mathrm{V}$ characteristic of the gated varactors versus gate voltage $\mathrm{V}_{\mathrm{g}}$ at various $\mathrm{V}_{\mathrm{d}} \cdot C_{o x}$ is the gate oxide capacitance and $C_{j o}$ is the zero-bias junction capacitance.

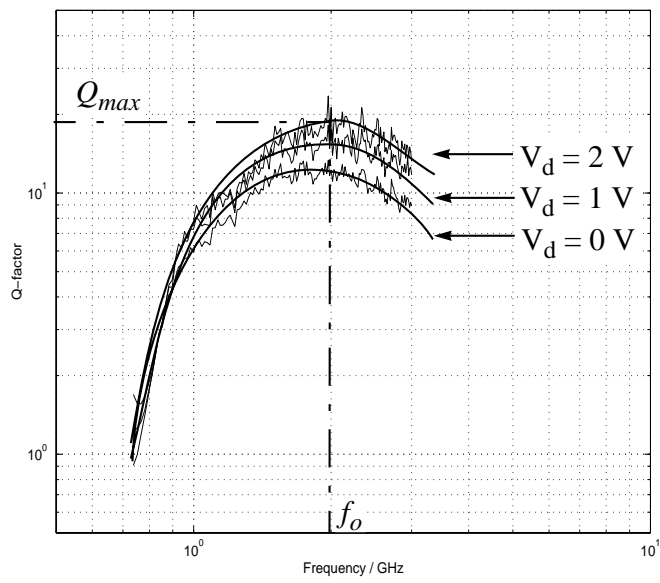

Fig. 4. Quality factor of the gated varactor versus frequency. A maximum quality of 22 is measured at an operating frequency of $2 \mathrm{GHz}$.

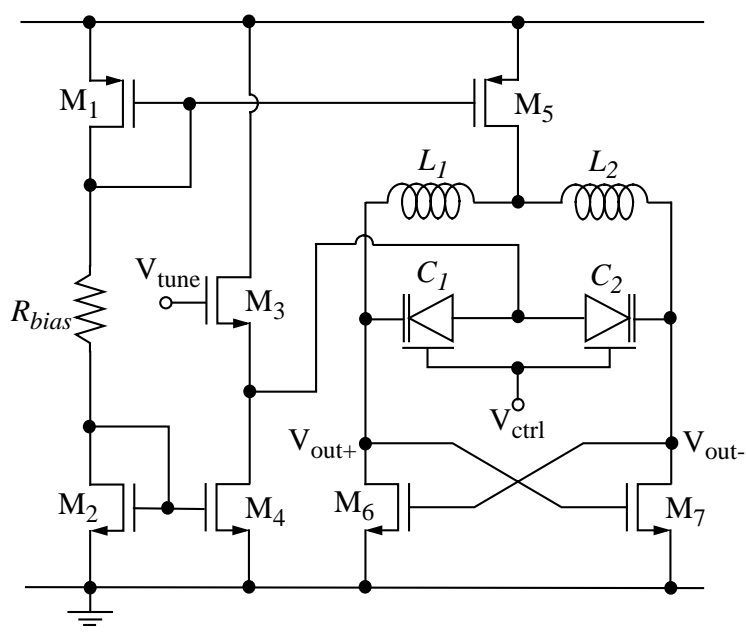

Fig. 5. Circuit diagram of the $2-\mathrm{GHz} \mathrm{VCO}$. Gate voltage of the gated varactors is applied to the node $\mathrm{V}_{\mathrm{ctrl}}$.

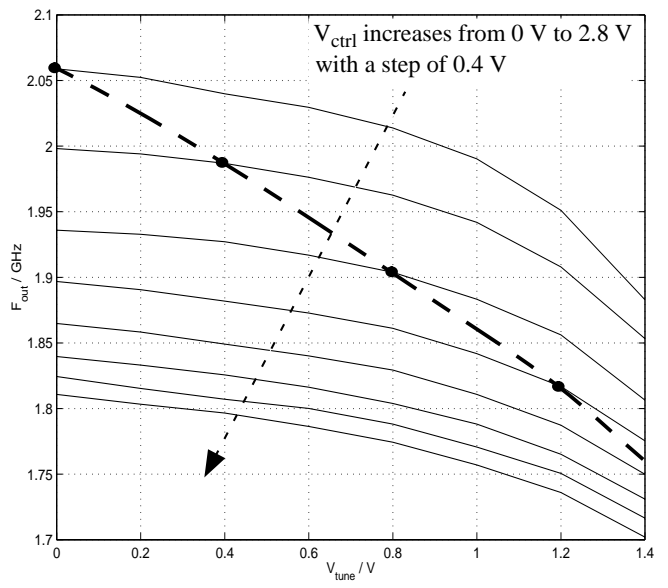

Fig. 6. Frequency tuning characteristic of the VCO. The dotted line shows the resultant tuning curve when $\mathrm{V}_{\text {ctrl }}$ and $\mathrm{V}_{\text {tune }}$ are tied together to form a single tuning node.

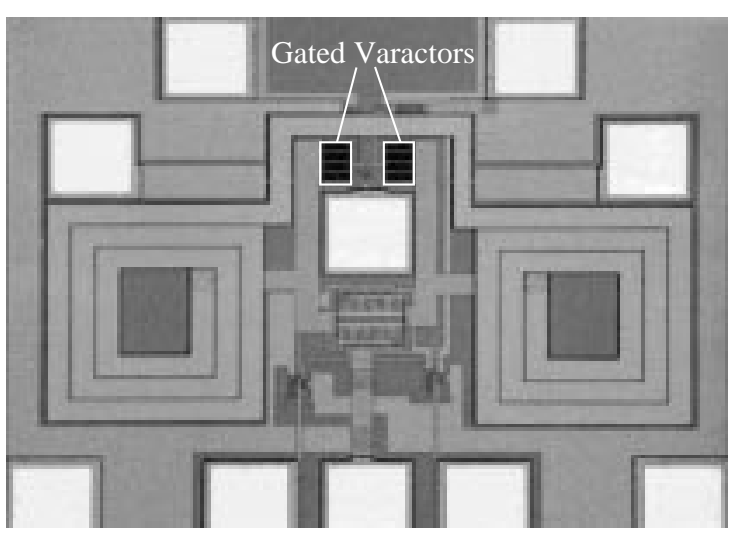

Fig. 7. Die photo of the 2-GHz VCO. The white boxes show the locations of the gated varactors which measure $45 \mu \mathrm{m}$ by $64 \mu \mathrm{m}$. 